\title{
Improving the SNR of Atomic Resolution STEM EELS \& EDX Mapping while Reducing Beam-damage by using Non-rigid Spectrum-image Averaging.
}

\author{
Lewys Jones ${ }^{1}$, Richard Beanland ${ }^{2}$ Sergio Lozano-Perez ${ }^{1}$, Karim Baba-kishi ${ }^{3}$ and Peter D. Nellist ${ }^{1}$ \\ 1. Department of Materials, University of Oxford, Oxford, UK \\ 2. Department of Physics, University of Warwick, Coventry, UK \\ 3. Department of Applied Physics, Hong Kong Polytechnic University, Hong Kong
}

The aberration corrected scanning transmission electron microscope (STEM) can now routinely image crystalline specimens at atomic resolution. Of the imaging modes available the relative ease of interpretation of the annular dark-field mode (ADF) makes this a popular choice, offering thickness / atomic-number type contrast. However, for composition studies either energy dispersive x-ray analysis (EDX) or electron energy-loss spectroscopy (EELS) are added to many STEMs. Unfortunately the achievable signal-noise ratio (SNR) of these signals significantly limits quantitative work.

There are three common approaches pursued to increase the SNR of elemental maps; increasing the beam-current, increasing the dwell-time or installation of a higher collection efficiency detector or spectrometer. However, the first two approaches have significant drawbacks. Increasing beam current can rapidly cause beam damage [1] or accelerate carbon contamination, while scanning slower additionally introduces scanning distortions, stage-drift or focal-drift into the frame [2]. Further, it is not always possible to increase beam-current (such as in monochromated systems), while increased dwelltimes make recording larger fields of view prohibitively time consuming for the operator.

In our new proposed method, instead of increasing either probe-current or dwell-time, signal is accumulated by recording multiple sequential spectral images. Using the simultaneously acquired ADF images, the data-cubes are then realigned and corrected for scanning-distortion using a custom non-rigid registration algorithm (available online as 'Smart Align' [3]). To test our proposed multi-frame approach two data-sets were recorded (Figure 1). The sample used was $\mathrm{Pb}_{2} \mathrm{ScTaO}_{6}$ prepared for STEM imaging by ion beam milling. This material exhibits incomplete long-range ordering on the Sc:Ta sublattice. Imaging was carried out near an anti-phase boundary. Simultaneous hardware synced spectra were recorded using a Gatan Quantum GIF and Oxford Instruments X-max $80 \mathrm{~mm}^{2}$ detector. A single $256 \times 256$ probe-position spectrum image was recorded with a dwell-time of $0.01 \mathrm{~s} / \mathrm{pix}$, and for comparison five separate $0.002 \mathrm{~s} /$ pix spectrum images (same total electron dose). The ADF images from the multi-frame acquisition were first realigned (rigid registered), cropped, then non-rigid-registered. Next, identical scan-distortion corrections were applied to the spectral data before being averaged to yield the restored spectra. To produce maps the EELS data were de-noised using principle component analysis before edge extraction. For the EDX maps characteristic x-rays were integrated after subtracting a linear background.

The ADF immediately shows the beam-damage and scanning-distortions which arise from using the slower scan speed. The fast-scanned, distortion-corrected, and averaged ADF image however shows improved SNR and straight lattice planes as expected. The location of the anti-phase boundary is now clearly resolved. The SNR of the Sc L-edge EELS maps show an improvement from 2.1 to 5.1 with the Sc positions relating directly with the ADF image. EDX maps are less clear but in the multi-frame average spectrum image the $\mathrm{Pb}$ lattice begins to become apparent. As sample damage is reduced so 
drastically, more frames could be recorded to improve SNR further if desired. This was not done here to allow a fair comparison at fixed total dose conditions

Our multi-frame spectrum image approach has demonstrated both reduced beam-damage and improved SNR for a fixed electron dose. This new approach allows for more beam-sensitive samples to be mapped, SNR to be increased and/or field-of-view and sampling to be improved [4].

\section{References:}

[1] R. Egerton, P. Li, and M. Malac, Micron 35 (2004), p. 399-409.

[2] L. Jones and P. D. Nellist, Microscopy \& Microanalysis. 19 (2013), p. 1050-1060.

[3] Demo code available free of charge for academic / non-commercial use at www.lewysjones.com .

[4] This research was supported by the European Union Grant Agreement 312483 - ESTEEM2.

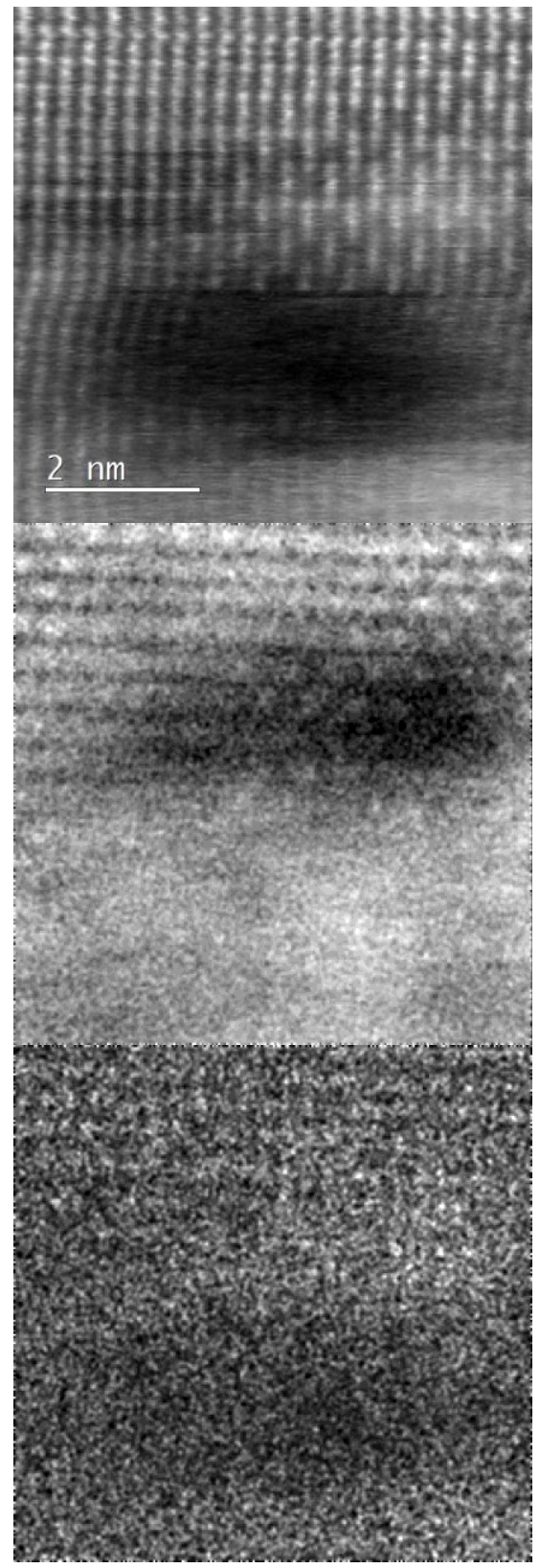

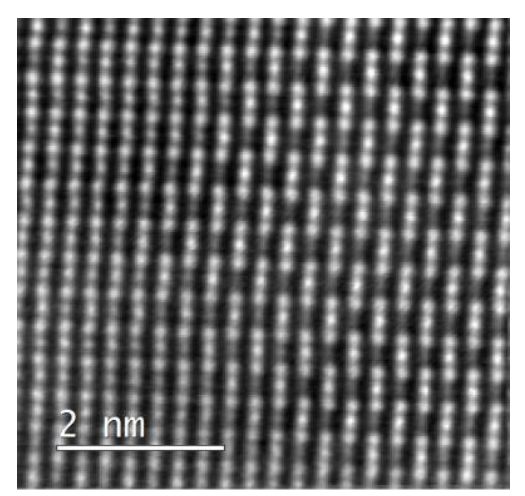
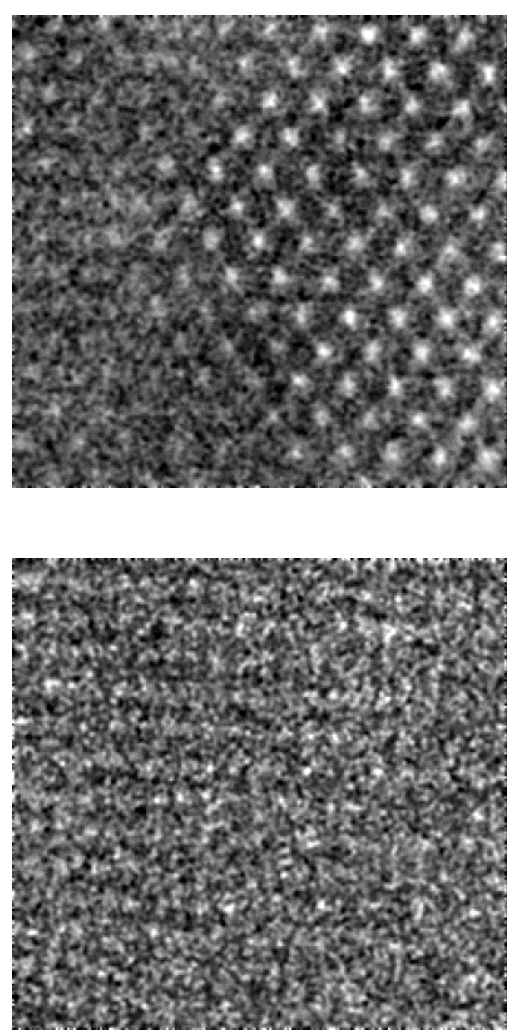

Figure 1. Conventional and proposed approaches to acquiring spectral image data. Example shows an anti-phase boundary region in a $\mathrm{Pb}_{2} \mathrm{ScTaO}_{6}$ perovskite.

The left column shows a single scan with $256 \times 256$ pixels at $0.01 \mathrm{~s} /$ pix. The right column shows the restored data from a set of 5 frames scanned at $0.002 \mathrm{~s} / \mathrm{pix}$ after non-rigid scandistortion compensation and averaging.

Rows show (top) the simultaneous ADF images, (middle) the EELS Sc L-edge map, and (bottom) the EDX $\mathrm{Pb} \mathrm{L} \alpha+\mathrm{M} \alpha$ map.

The slightly smaller field of view $(225 \times 214$ pix $)$ is due to cropping after drift realignment. Both data were recorded at $30 \mathrm{M}$ magnification. 